\title{
IL-33 Attenuates Sepsis by Inhibiting IL-17 Receptor Signaling through Upregulation of SOCS3
}

\author{
Ran Lv Jinning Zhao Min Lei Dongju Xiao Yijin Yu Junran Xie \\ Department of Anesthesiology, Sir Run Run Shaw Hospital, School of Medicine, Zhejiang University, \\ Zhejiang, China
}

\section{Key Words}

Sepsis $\bullet \mathrm{CLP} \cdot \mathrm{IL}-33 \cdot \mathrm{IL}-17 \cdot \mathrm{SOCS} 3$

\begin{abstract}
Background/Aims: Sepsis is a systemic inflammatory response during infection. There are limited therapeutic options for sepsis patients. Interleukin (IL)-33 has been reported recently with a beneficial effect in mouse sepsis. Methods: In this study, we initiated a clinical study to measure serum levels of pro-inflammatory cytokines including IL-33 in sepsis patients. Next, we employed cecal ligation and puncture (CLP) to study the role of IL-33 during sepsis. To further dissect the molecular mechanism, we used in vivo knockout models and in vitro knockdown murine embryonic fibroblasts (MEFs) to investigate the cross-talk between IL33 and IL-17 signaling, and to identify the potential downstream mediators. Results: IL33 and IL-17 were upregulated in both clinical and experimental sepsis. In CLP, IL-33 (-/-) mice showed higher mortality rate, and IL-33 treatment improved the survival rate. Elevated proinflammatory cytokines in sepsis were related to IL-17 from $\gamma \delta T$ cells. IL-33 treatment suppressed production of these cytokines by targeting IL-17 signaling both in vivo and in vitro. Finally, IL-33 was shown to inhibit the IL-17 pathway via activating suppressor of cytokine signaling (SOCS)-3. Conclusion: Collectively, the results suggest that IL-33 plays a negative regulatory role in sepsis progression by inhibiting IL-17 pathway through activating SOCS3. This finding would inspire a new therapeutic strategy for treating sepsis.
\end{abstract}

(C) 2017 The Author(s)

Published by S. Karger AG, Basel

\section{Introduction}

Sepsis is a systemic inflammatory response to pathogenic infections, which develops when the initial host response fails to control the infection, resulting in widespread inflammations in multiple organs [1]. During the onset of sepsis, the inflammatory system 
becomes hyperactive, and produces pro-inflammatory cytokines and chemokines such as tumor necrosis factor- $\alpha$ (TNF- $\alpha$ ), interleukin (IL)-6, IL-1 and IL-8 [2]. Simultaneously, activations of the coagulation and complement systems result in excessive productions of proinflammatory mediators such as C5a. Phagocytic cells, such as neutrophils and macrophages, respond to many of these mediators by releasing granular enzymes and producing reactive oxygen species (ROS) such as H2O2 [3], as a result, tissue damages and organ failure occur [4]. Various strategies in clinical sepsis trials involve blocking specific inflammatory mediators such as TNF- $\alpha$ activated protein C (APC), macrophage migration inhibitory factor (MIF) and the complement split product C5a. Targeting APC has recently been approved for treatment of sepsis [5]. However, most clinical trials, Therefore, advances in unraveling the molecular basis for the host response to sepsis are needed for new intervention strategies to reduce mortality in sepsis patients.

IL-33 is a member of the IL-1 family cytokines [6], and is normally produced by damaged or necrotic barrier cells. Activation of IL-33 signaling involves its binding to the heterodimeric receptor complex consisting of ST2 (IL-1RL1) and IL-1 receptor accessory protein (IL-1RAcP), which subsequently recruits signaling adaptor MyD88, kinases IRAK1 and IRAK4, and TRAF6. Such molecular cluster in the cytoplasm leads to activation of nuclear factor- $\kappa \mathrm{B}(\mathrm{NF \kappa B})$ and mitogen-activated protein kinases ERK, p38 and JNK, which promote cell proliferation, survival and cytokine secretions [7]. IL-33 receptor is widely expressed in many cell types including Th2 helper cells, regulatory $\mathrm{T}$ cells, $\mathrm{CD}^{+} \mathrm{T}$ cells and natural killer cells, suggesting IL-33 signaling has a pleiotropic role in tissues. For instance, IL-33 protects the host against helminth infection. In contrast, IL-33 also promotes the pathogenesis of asthma by expanding Th2 cells, and mediates anaphylaxis by mast cell activation $[8,9]$. Recent study has shown IL-33 could attenuate sepsis by enhancing neutrophil influx to the site of infection in a mouse experimental sepsis model [10], suggesting a therapeutic potential for IL-33 in sepsis.

On the other hand, IL-17 is a pro-inflammatory cytokine, which was first identified as a T cell-derived molecule to activate IL-6 production [11]. IL-17 secretion is found in multiple cell types including T helper 17 (Th17) cells, NK cell, $\gamma \delta$ T cells, mast cells and Th2 cells. Preclinical and clinical studies have linked IL-17 to the pathogenesis of inflammatory diseases. For instance, abnormal expression of IL-17 can cause damages in multiple tissues, such as the lung and the liver [12]. Therefore, understanding the regulation of IL-17 signaling will provide us with more choices to block its activity.

Suppressor of cytokine signaling (SOCS) proteins are negative regulators for various cytokines via inhibiting the JAK/STAT pathway [13]. 8 members, including SOCS-1 to SOCS7 and CIS, have been identified in this family. SOCS proteins can be induced by various cytokines and Toll-like receptor (TLR) ligands. SOCS3 has been demonstrated to play a regulatory role in signaling downstream of a wide range of cytokine receptors, as well as in TLR-mediated host defense $[14,15]$. TLR signaling plays a significant role in mounting systemic responses to invading pathogens and subsequent development of sepsis. SOCS3 is implicated in regulating inflammatory response and controlling tissue damage during sepsis [16].

In this study, we sought to investigate the therapeutic potential of IL-33 in sepsis. We first analyzed IL-33 serum levels in clinical and experimental sepsis models. Then we evaluated the protective role of IL-33 in cecal ligation and puncture (CLP) with mouse knockout model. Meanwhile, we showed that elevated pro-inflammatory cytokines in sepsis model were related to IL-17 signaling, and $\gamma \delta$ T cells were the major source of IL-17. Subsequently, we confirmed that IL-33 attenuated sepsis by suppressing IL-17-induced pro-inflammatory cytokine productions both in vivo and in vitro. Finally, we showed that IL-33 inhibited IL-17 pathway by regulating SOCS3 activation. 


\section{Cellular Physiology Cell Physiol Biochem 2017;42:1961-1972

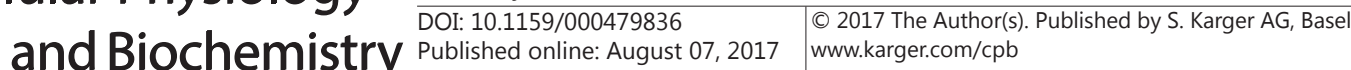

Lv et al.: New Therapeutic Strategy for Sepsis

\section{Materials and Methods}

Study design

Ten healthy volunteers and ten patients with systemic inflammatory response syndrome (SIRS) were enrolled as healthy control and Sepsis groups in Sir Run Run Shaw Hospital. All ten patients had pathological evidence suggestive of gram-negative infection and at least two of clinical manifestations based on SIRS guideline [17]. No patients showed signs of hypoperfution or organ dysfuntion. The experimental protocol was approved by the institutional ethical review board. All subjects were informed of the risks and purposes of the study before their written consent was obtained.

\section{Animal experiment}

All animal experiments were approved by the institutional animal care and use Committee. IL-33 $(-/-), \operatorname{TCR} \beta(-/-)$ and IL-33 (-/-) TCR $\beta$ (-/-) knockout strains were on C57BL/6 background and were obtained from The Jackson Laboratory (San Diego, CA). All mice used in the study have been genotyped and verified for the correct gene knockout. Mice at $6 \sim 8$ weeks old, were subjected to Cecal Ligation and Puncture (CLP) and processed for further analysis in accordance with the guidelines provided by university animal center.

\section{Cecal ligation and puncture}

We anesthetized the mice and shaved the abdominal wall. After midline laparotomy, we exposed the cecum, ligated below the ileocecal valve without causing intestinal obstruction and then punctured twice with a $21 \mathrm{G}$ needle. In some experiments, we used a $30 \mathrm{G}$ needle to induce a mild CLP. We assessed clinical score every $12 \mathrm{~h}$ after CLP, as previously described. The symptoms of the maximum score of six are lethargy, piloerection, tremors, periorbital exudates, respiratory distress and diarrhea. Each condition was scored as 1 . Mice with a clinical score of $>1$ were defined as showing signs of sepsis. We determined survival rate daily for $7 \mathrm{~d}$ after CLP [10].

\section{Cytokines measurement}

The blood samples were drawn from human subjects in accordance with institutional ethical review guidelines. In CLP experiment, the blood samples were collected from mice in CLP experiment in accordance with guidelines approved by institutional animal care and use committee. The serum levels of the indicated cytokines were determined by ELISA using matched pairs of antibodies from R\&D Systems according to the manufacturer's recommendations. Absorbance was read on a Multiscan FC plate reader and analyzed with SkanIt for Multiscan FC software (Thermo Scientific, Waltham, MA, USA). The cytokine measurements for each sample subject were performed in technical repeat for at least three times.

\section{Experimental materials and cell culture}

Dulbecco's modified Eagle's medium (DMEM), penicillin, streptomycin and trypsin were supplied by Gibco (Thermo Fisher). Fetal bovine serum (FBS) was supplied from Hyclone (GE Healthcare, Little Chalfont, Buckinghamshire, United Kingdom). Primary culture of mouse embryonic fibroblasts (MEFs) was based on the method described previously [18]. Recombinant IL-17 and IL-33 for treatment were obtained from InvivoGen. Anti-TAK1 (\#4505) and anti-phosphor-IкB $\alpha$ (\#9246) were purchased from Cell Signaling Technology. Anti-SOCS3 (ab16030), anti-IKKß (ab32135) and anti-JNK2 (ab76125) antibodies were purchased from Abcam. Anti-phosphor-JNK2 antibody was obtained from Novus Biologicals.

\section{Realtime PCR analysis}

Total RNA was extracted from one million cells using TRIzol solution (Invitrogen, Carlsbad, CA, USA). $1 \mu \mathrm{g}$ of total RNA was reverse transcribed into cDNA for $1 \mathrm{~h}$ at 50 degree using oligo dT primer and reverse transcriptase in the presence of RNAse inhibitor. Transcribed cDNA template (50 ng) was incubated with $200 \mathrm{nM}$ target gene primers or GAPDH primers (normalization control) in a total volume of $20 \mu \mathrm{l}$ using KAPA SYBR FAST qPCR kit. The pairs of primers for each target cytokine are shown below:

CCL20: TGTACGAGAGGCAACAGTCG; TCTGCTCTTCCTTGCTTTGG

CXCL1: CTTGACCCTGAAGCTCCCTT; AGGTGCCATCAGAGCAGTCT

CXCL2: AAAGTTTGCCTTGACCCTGA; TCCAGGTCAGTTAGCCTTGC 
CXCL9: TAGGCAGGTTTGATCTCCGT; CGATCCACTACAAATCCCTCA
CXCL10: CCTATGGCCCTCATTCTCAC; CTCATCCTGCTGGGTCTGAG
IL-6: CACAGAGGATACCACTCCCAACA; TCCACGATTTCCCAGAGAACA

Western blot analysis

After treatment, the cells were washed once in PBS and harvested in RIPA lysis buffer. The protein concentration was measure with protein BCA protein assay. $40 \mu \mathrm{g}$ protein lysate was prepared in SDS sample buffer and separated on an 8-12\% SDS-polyacrylamide gel and then transferred to a PVDF membrane. Membranes were blocked with 5\% non-fat milk and then incubated with indicated primary antibody in 5\% non-fat milk prepared for over-night at 4 degree. After washed with TBS-T, the membranes were incubated with the corresponding secondary antibodies coupled to HRP (Horse Radish Peroxidase). After washed with TBS-T again, western signals were visualized using Western Pico Super ECL reagent (Pierce, WI, USA). The results shown here were the representatives of at least three times of independent experiments.

Flow cytometry cell analysis

Flow cytometric analysis: Leukocytes were stained with the appropriate concentration of the following antibodies: PE/FITC TCR $\delta$ chain (GL3), FITC V 44 TCR (UC3-10A6), FITC V84 TCR (GL2). For intracellular cytokine staining, cells were pre-incubated for $4 \mathrm{~h}$ with PMA $(20 \mathrm{ng} / \mathrm{ml})$, ionomycin $(500 \mathrm{ng} /$ $\mathrm{ml})$ and brefeldin $\mathrm{A}(10 \mu \mathrm{g} / \mathrm{ml})$ at $37^{\circ} \mathrm{C}$ and $5 \% \mathrm{CO}_{2}$. All the drugs were purchased from Sigma Aldrich. After surface marker staining, cells were fixed, permeabilized and stained with anti- interferon (IFN)- $\gamma$, and antiIL-17 antibodies (BD Pharmingen, San Diego, CA, USA). Cells were acquired by FACScalibur flow cytometer (Becton Dickinson, USA) and analyzed either by Cell Quest or FlowJo software.

Lentiviral infection mediated gene knockdown

Lentiviral particles were produced by transfection of HEK293 cells (by the calcium-phosphate method) with a pGIPZ lentiviral vector (GE Dharmacon) encoding either a non-silencing short hairpin RNA or SOCS3 short hairpin RNAs, along with the packaging vectors psPAX2 and pMD2. MEFs were infected with the lentiviruses for $8 \mathrm{~h}$. After $72 \mathrm{~h}$, the infected cells were enriched by FACS (based on GFP expression) and subsequently used for experiments.

Statistical analysis

All experimental data were presented as mean \pm standard deviation (SD). Statistical differences were analyzed by one or two way ANOVA analysis followed by a Tukey's post hoc test. A p value $<0.05$ was considered significant

\section{Results}

To understand the physiological role of IL-33 in sepsis, we first measured its blood concentration in patients with systemic inflammatory response syndrome (SIRS). We recruited 10 patients with pathological evidence of infection and sepsis symptoms in accordance to the SIRS guideline [17], though they didn't manifest multiple organ dysfunction syndrome. We enrolled another 10 healthy volunteers as the control group. Eligibility was assessed through authorized medical record review, and all eligible subjects provided informed consent for the study. In addition to IL-33, we included another three key proinflammatory cytokines or mediators IL-17, IL- 6 and CXCL1 as readouts for the comparison study. As shown in Fig. 1a-1d, subjects from the sepsis group showed significantly higher serum levels of all four cytokines, indicating their elevated systemic inflammatory status. To corroborate this observation, we measured IL-33, IL-17, IL-6 and CXCL1 expressions in CLP mouse model for clinical sepsis. C57BL/6 mice were given a milder form of CLP and their blood was collected for cytokine measurements on different days after CLP (Fig. 1e-1h). In accordance with what we observed in patient samples, CLP mice produced significantly higher levels of IL-33, IL-17, IL-6 and CXCL1. The serum levels of all four cytokines increased steadily from day 1 to day 7 after CLP. In conclusion, IL-33, IL-17, IL-6 and CXCL1 were induced in both clinical and experimental models.

\section{KARGER}


Fig. 1. The level of IL-33, IL-17, IL-6 and CXCL1 were determined by sandwich ELISA. (a-d) Ten healthy volunteers and ten patients with systemic inflammatory response syndrome (SIRS) were enrolled as healthy control and sepsis groups (one way ANOVA analysis followed by a Tukey's post hoc test, ** $\mathrm{p}<0.01)$. (e-h) The subjects were given a milder form of CLP and the serum levels of IL-33, IL-17, IL-6 and CXCL1 were measured by ELISA. All data represent mean $\pm \mathrm{sd}, \mathrm{n}=10$ mice per group.

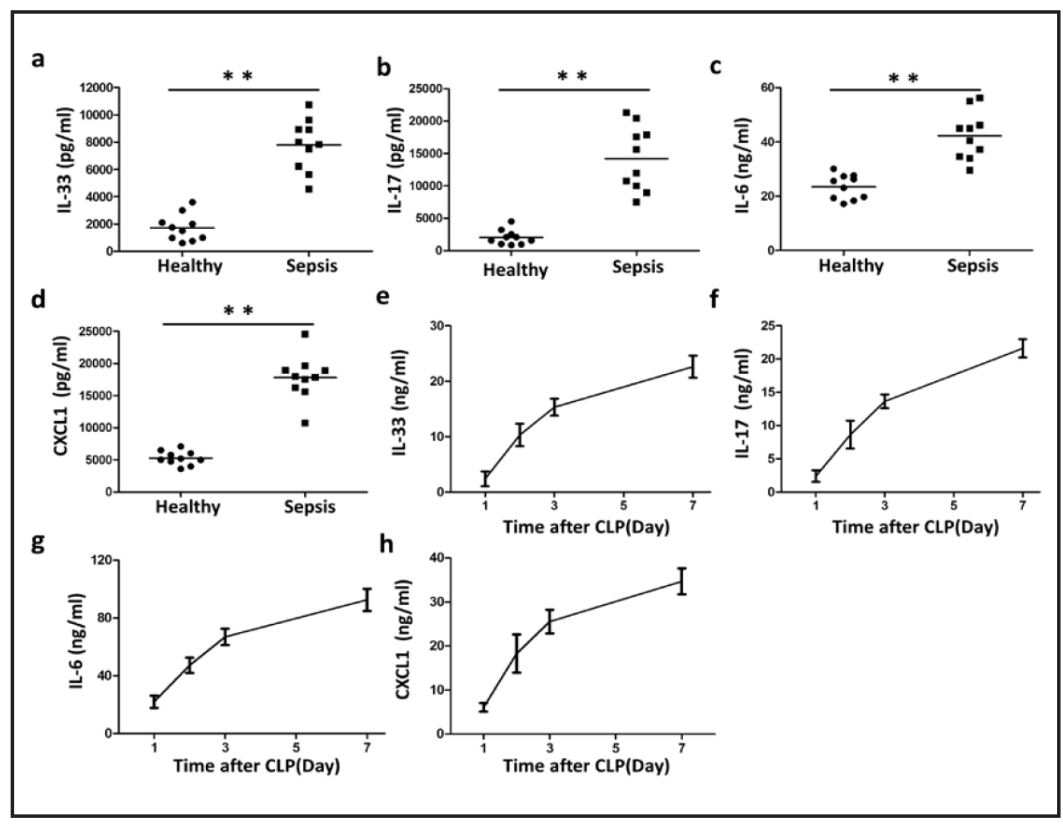

Fig. 2. Neutralizing IL-17 protects IL-33 (-/-) mice in CLP sepsis model. (a) Survival rate of $\mathrm{C} 57 \mathrm{BL} / 6$ wild type and IL-33 (-/-) mice in CLP model $(n=10$ per group). (b) Survival rate of C57BL/ 6 wild type and IL-33 (-/-) mice treated with anti-IL-17 antibody $(50 \mu \mathrm{g})$ in CLP model ( $\mathrm{n}=$ 10 per group). (c) The level of IL-17 was determined by sandwich ELISA after 2 days of CLP.

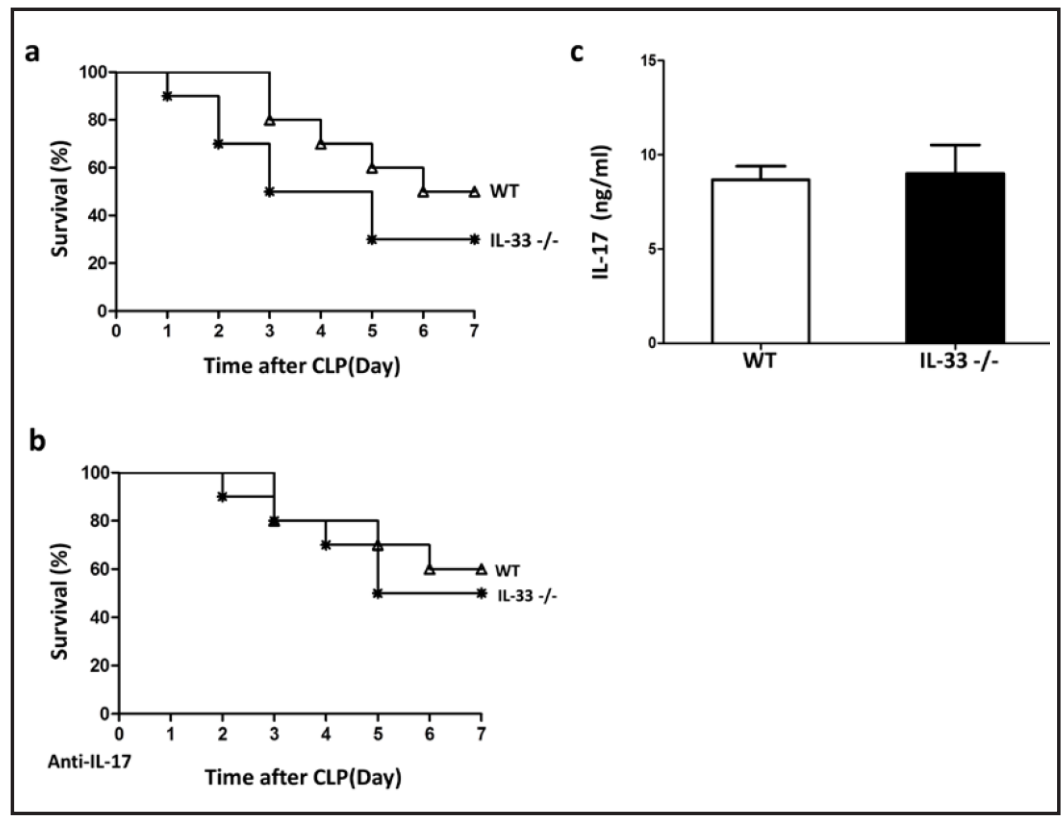

Previous study has reported that IL-33-treated mice exhibited reduced mortality in CLP sepsis model [10]. To gain further insight into the role of endogenous IL-33 in sepsis, we chose to study the survival rate of IL-33 deficient mice in experimental sepsis model. As shown in Fig. 2a, IL-33 deficient mice exhibited higher mortality rate than that of wild type (WT) mice when challenged with a mile form of CLP. Nearly $50 \%$ of WT mice survived till day 7 after CLP, whereas only around 30\% of IL-33 (-/-) mice did so. IL-17 and its receptor pathways play important roles in pathological process of sepsis [19]. Abnormal expression of IL-17 during pathogen infection may contribute to tissue inflammation resulting in high mortality $[20,21]$. Therefore, we next examined the protective effect of neutralizing IL-17 in the CLP sepsis model. WT and IL-33 (-/-) mice were both treated with anti-IL-17 antibody through intravenous injection before CLP. Their mortality rates were monitored for 7 days. As shown in Fig. $2 \mathrm{a}$ and $2 \mathrm{~b}$, neutralizing IL-17 reduced the mortality rate in IL-33 (-/-) mice from $70 \%$ to $50 \%$. There was also a slight improvement in the survival rate of WT mice 
Fig. 3. Interleukin-33 attenuates sepsis by suppressing the level of IL-17 related cytokines. (a) Survival rate of C57BL/6 mice ( $\mathrm{n}=5$ per group) treated daily with $1,10 \mu \mathrm{g} / \mathrm{ml}$ of IL-33 protein or PBS (WT) for 7 days and blood was collected. (b) IL6, CXCL1, CXCL2, CXCL9, CXCL10, CCL20 concentrations were measured by ELISA. The results shown are from three independent experiments (one way ANOVA analysis followed by a Tukey's post hoc test, ${ }^{*} \mathrm{p}<0.05$; ** $<0.01$ ).

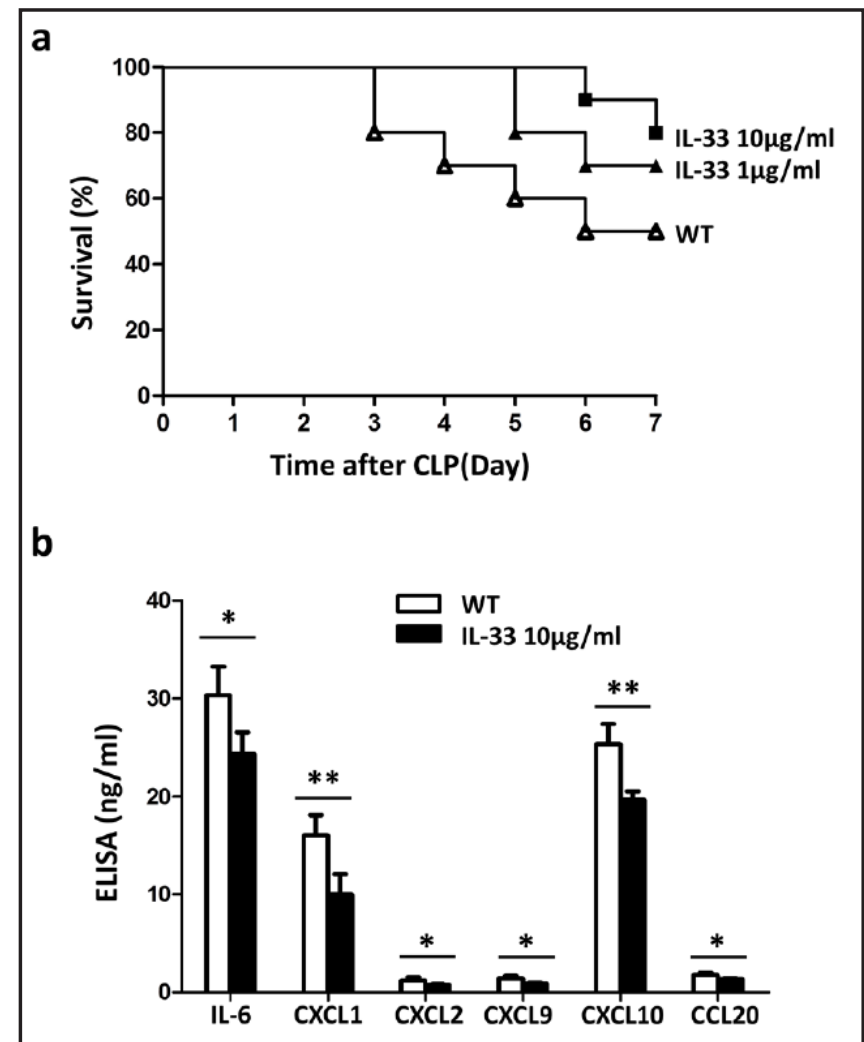

treated with anti-IL-17 after CLP. Next, we proceeded to measure IL-17 levels in mice 2 days after CLP. However, there was no significant difference between WT and IL-33 (-/-) mice (Fig. 2c), suggesting the higher mortality found in IL-33 deficient mice after CLP treatment was not due to abnormal IL-17 expression. Given that neutralizing IL-17 improved sepsis survival rate in IL-33 (-/-) mice, it was likely that IL-33 deficiency affected IL-17 downstream signaling instead of cytokine biogenesis per se.

Since IL-33 deficiency affected mice survival rate after CLP treatment, we next examined whether IL-33 injection protected mice against sepsis. Mice were injected intravenously with recombinant IL-33 before CLP. As shown in Fig. 3a, IL-33 treatment markedly reduced sepsisassociated mortality rate. Mice without treatment showed $\sim 50 \%$ survival rate on day 7 after CLP. Compared to untreated control, mice treated with either $1 \mu \mathrm{g}$ or $10 \mu \mathrm{g}$ IL-33 exhibited $\sim 70 \%$ or $\sim 80 \%$ survival rates, respectively. The protection was positively correlated to the injection dose of IL-33, consistent with previous studies $[10,22]$. To further study how IL-33 treatment conferred the protection against sepsis, we analyzed pro-inflammatory cytokines that could mediate CLP-induced inflammation. We hypothesized that IL-33 might attenuate the pro-inflammatory cytokine expressions to contain the CLP-induced inflammation. In addition, IL-17 was shown to reduce sepsis mortality in IL-33 deficiency mice (Fig. 2). Therefore we measured a panel of pro-inflammatory cytokines, including IL-6, CXCL1, CXCL2, CXCL9, CXCL10 and CCL20, all of which are also regulated by IL-17/receptor pathway [23]. WT mice were treated with $10 \mu \mathrm{g}$ IL-33 before CLP. After 2 days, the serum levels of these cytokines were determined by ELISA. In line with our hypothesis, IL-33 treatment markedly reduced the serum concentrations of these IL-17 regulated pro-inflammatory cytokines, as compared to controls (Fig. 3b). This result further implied that IL-33 might have an undetermined role in regulating IL17 pathway in experimental sepsis model.

In addition to the Th17 cells, several other cell types including $\gamma \delta \mathrm{T}$ and $\mathrm{CD} 8^{+}$also produce IL-17 [24-26]. In order to identify the early source of IL-17 during sepsis, we performed CLP experiment on TCR $\beta(-/-)$ mice that were defective in $\gamma \delta$ T cell generation. 7 days after CLP, TCR $\beta(-/-)$ mice exhibited $\sim 40 \%$ survival rate. Knocking out IL-33 in this background 

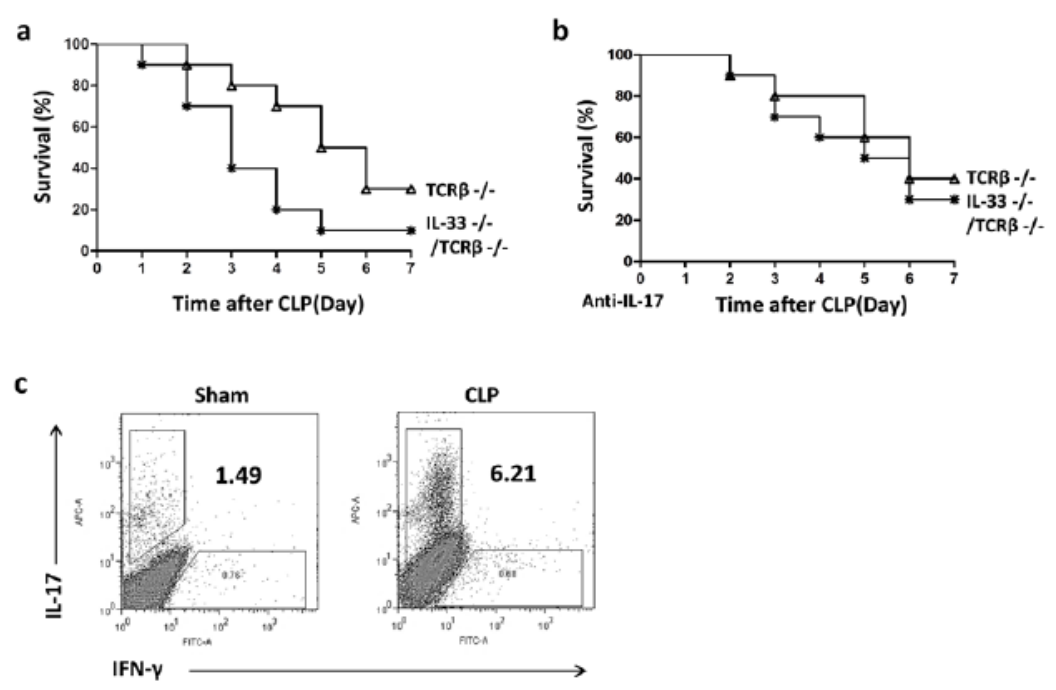

Fig. 4. $\gamma \delta$ T cells were source of IL-17 in CLP sepsis model. (a) Survival rate of TCR $\beta(-/-)$ and IL-33 (-/-)/ TCR $\beta$ (-/-) mice in CLP model (n = 10 per group). (b) Survival rate of TCR $\beta$ (-/-) and IL-33 (-/-)/TCR $\beta$ $(-/-)$ mice treated with anti-IL-17 antibody $(50 \mu \mathrm{g})$ in CLP model (n = 10 per group). (c) Percentage of IL17 ${ }^{+}$ $\mathrm{TCR} \gamma \delta^{+}$cells in TCR $\beta^{-}$mice with CLP or sham-operated mice (sham).

further reduced the survival rate to $\sim 15 \%$ (Fig. 4 a). In addition, IL-17 treatment in TCR $\beta$ $(-/-) /$ IL-33 (-/-) double knockout mice alleviated the mortality rate (Fig. $4 \mathrm{~b})$, suggesting IL17 production was still intact in TCR $\beta(-/-)$ mice and was probably mediated through $\gamma \delta \mathrm{T}$ cells. To further validate this, we measured IL-17 production in $\gamma \delta$ T cells in CLP experiment. $\gamma \delta$ T cells were isolated from TCR $\beta(-/-)$ mice challenged with CLP. The percentage of $\gamma \delta$ T cells expressing IL-17 and IFN $\gamma$ was measured in flow cytometry. As shown in Fig. 4c, CLP treatment markedly increased IL-17+/IFN $\gamma+\gamma \delta$ T cells from $1.49 \%$ (sham operated group) to $6.21 \%$. Taken together, $\gamma \delta \mathrm{T}$ cell was one of the sources that produced IL-17 during pathological process of sepsis.

We showed that, after CLP, IL-33 suppressed the expressions of IL-6, Cxcl1, Cxcl2, Cxcl9, Cxcl10 and Ccl20, which were reported to be regulated by IL-17 [24]. Next, we asked whether IL-33 could directly attenuate their expressions in response to IL-17 treatment in vitro. MEFs from WT mice were treated with $10 \mu \mathrm{g} / \mathrm{ml}$ IL-33 or PBS as control for 12 hours before IL-17 treatment. 12 hours after IL-17 stimulation, the cells were harvested to measure IL-6, Cxcl1, Cxcl2, Cxcl9, Cxcl10 and Ccl20 mRNA expression levels. As shown in Fig. 5, IL-17 greatly activated the gene expressions of these cytokines in vitro. In comparison, IL33 pre-treatment significantly suppressed their expressions in response to IL-17. Therefore, this in vitro result further supported that IL-33 could downregulate IL-17 signaling pathway.

Finally, we studied how IL-33 suppressed IL-17-induced cytokine expressions. Upon IL-17 stimulation, IL-17RA and IL-17RC form a heterodimeric complex to recruit Act1 and TRAF6, which is a signaling E3 ligase and subsequently activates a pivotal kinase TAK1. TAK1 activation can eventually lead to the activation of several downstream signaling factors, including I $\kappa \mathrm{B} \alpha$ kinase (IKK) and its target transcription factor, the two major MAP kinases JNK and p38 and their downstream transcription factor C/EBP, which act together to induce pro-inflammatory cytokines and chemokines $[23,24]$. Therefore, we stimulated MEFs with IL-17 for $30 \mathrm{~min}$ and $60 \mathrm{~min}$ in the presence or absence of IL-33, and measured the activation

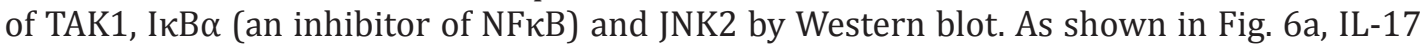

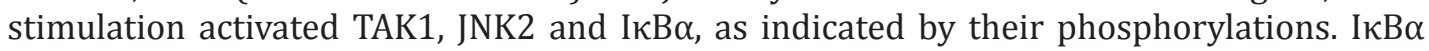
phosphorylation leads to its own degradation that releases NFKB for nuclear translocation and gene transcription. Their phosphorylations in MEFs could be detected as early as $30 \mathrm{~min}$ after IL-17 stimulation. Interestingly, in the presence of IL-33, IL-17-mediated activation of 
Fig. 5. The level of Il6, Cxcl1, $\mathrm{Cxcl} 2, \mathrm{Cxcl} 9$, Cxcl10 and $\mathrm{Ccl} 20$ concentrations were measured by q-PCR. (a-f) MEFs were treated 12 hours with $10 \mu \mathrm{g} / \mathrm{ml}$ of IL-33 protein or untreated (WT) before q-PCR. The results shown are from three independent experiments (one way ANOVA analysis followed by a Tukey's post hoc test, ${ }^{*} \mathrm{p}<0.05 ;{ }^{* *} \mathrm{p}<0.01$ ).

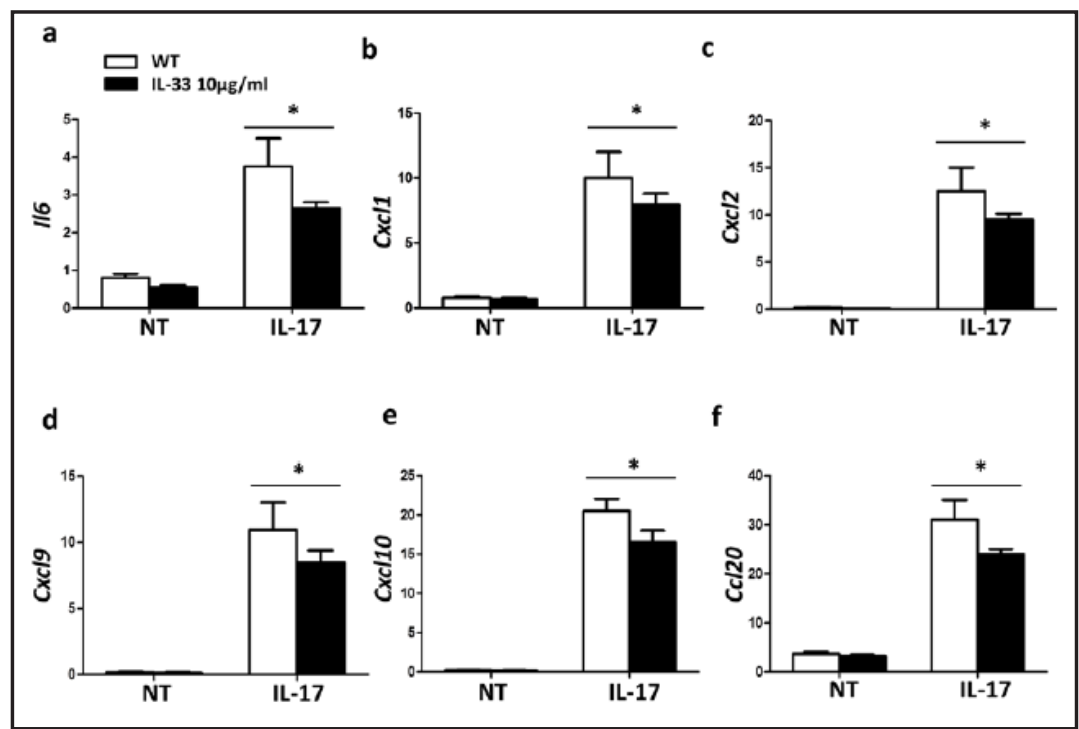

b

a
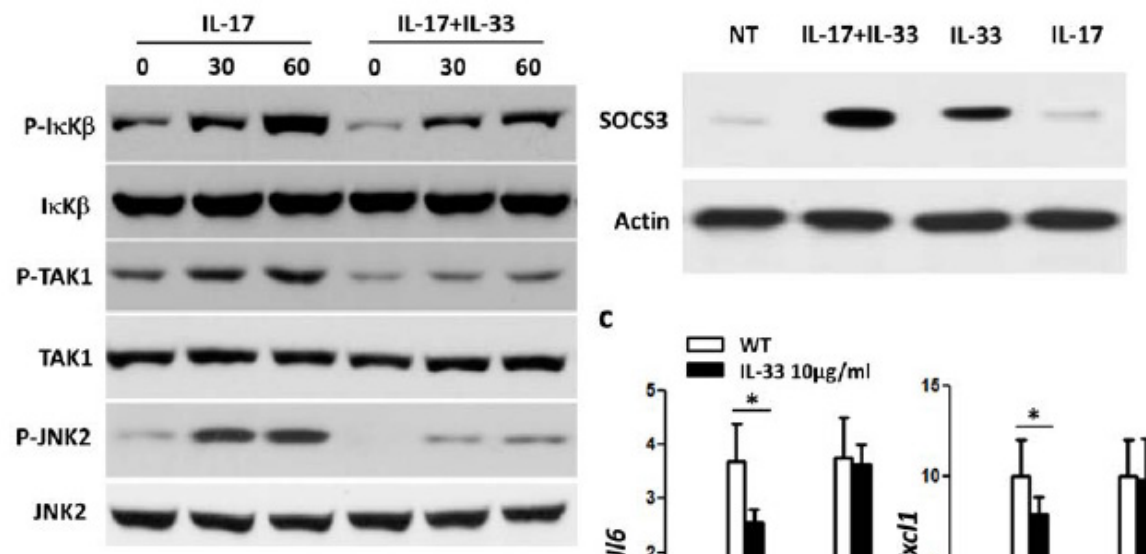

NT IL-17+IL-33 IL-33 IL-17

c

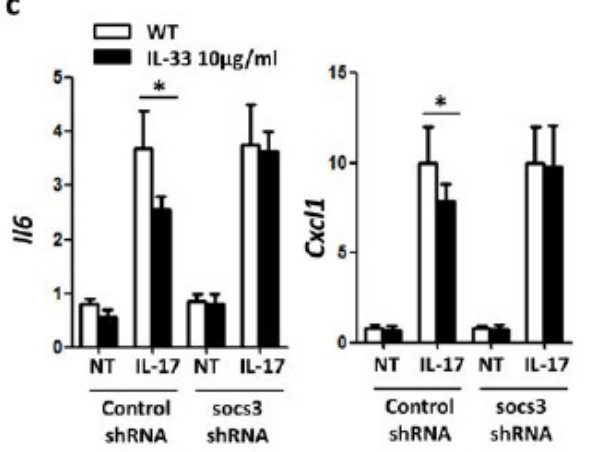

Fig. 6. IL-33 inhibited IL-17 pathway by upregulation of SOCS3. (a) IB analyses of the indicated phosphorylated (P-) and total proteins in MEFs with multiple inductors for the indicated time periods; (b) SOCS3 expressed in MEFs with multiple inductors for $30 \mathrm{~min}$; (c) The level of Il-6, Cxcl1 concentrations were downregulated by IL-33, but rescued by socs3 shRNA(q-PCR). MEFs had been transduced with a GFP-expressing lentiviral vector (pGIPZ) encoding a control shRNA or a socs3 shRNA. MEFs were treated 12 hours with $10 \mu \mathrm{g} / \mathrm{ml}$ of IL-33 protein or untreated (WT) and then stimulated with or without IL-17 for 30 min before q-PCR. The results shown are from three independent experiments (two way ANOVA analysis followed by a Tukey's post hoc test, $\left.{ }^{*} \mathrm{p}<0.05\right)$.

TAK1, JNK2 and IкB $\alpha$ were significantly attenuated, consistent with the notion that IL-33 suppressed IL-17-mediated cytokine productions. Since TAK1 was upstream of NFKB and JNK2, this result suggested IL-33 signaling might act on TAK1 or its upstream regulators to suppress IL-17-mediated pathway activation.

Suppressor of SOCS3 has been identified as negative feedback inhibitor for various TLRs and cytokine signaling. Next, we asked whether IL-33 could regulate IL-17 signaling 
via SOCS3. As a result, IL-33 was found to induce SOCS3 in MEFs, whereas IL-17 alone failed to do so (Fig. 6b). Interestingly, MEFs pretreated with IL-33 showed profound SOCS3 induction in response to IL-17 stimulation, suggesting IL-33 may downregulate IL-17 pathway via SOCS3 signaling. To further confirm this, we also evaluated the role of SOCS3 in pro-inflammatory cytokine productions. We transfected MEFs with either control shRNA or SOCS3 shRNA, and challenged these cells with IL-17 in the presence or absence of IL-33, followed by measuring IL-6 and Cxcl1 expressions. As shown in Fig. 6c, IL-17 enhanced both IL- 6 and Cxcl1 expressions in control MEFs, and such induction could be suppressed by IL33. However, in SOCS3 knock-down MEFs, IL-17-mediated IL-6 and Cxcl1 inductions were not affected by IL-33 treatment. Taken together, IL-33 attenuated IL-17 receptor signaling through SOCS3 induction.

\section{Discussion}

Sepsis is one of the most common causes of death in hospitalized patients, with mortality rates between $30 \%$ and $70 \%$, and affecting over 18 million people worldwide. Pathogenesis of severe sepsis involves an overwhelming production of pro-inflammatory cytokines such as TNF $\alpha$, IL- $1 \beta$ and IL-6, which are generally considered as the mediators for SIRS, leading to tissue or organ damage [1]. Therefore, most current therapeutic strategies have targeted on these mediators using monoclonal antibodies against TNF $\alpha$, IL-1-receptor antagonists and TNF-receptor fusion proteins. Unfortunately, most of these strategies did not meet the expectation in large, multicenter clinical trials. Therefore, to further understand the pathogenesis of sepsis and explore the novel mediators as intervention targets, the use of experimental sepsis model have become necessary to improve the treatment outcome in clinical trials [1, 3, 27-29].

IL-33 is a functional ligand for ST2. IL-33/ST2 signaling was first described in T-cell mediated immune response during acquired immune response [6]. A recent study has also revealed that IL-33 is important for innate-type mucosal immunity in the lungs and guts [30]. Furthermore, IL-33 was shown to prevent apoptosis of T lymphocytes and improve survival rate of CLP-induced sepsis [22], supporting the role of IL-33 and its associated signaling molecules as novel intervention targets in sepsis therapy. In order to validate this, we evaluated IL-33 serum levels in 10 patients with pathological evidence of infection and sepsis symptoms in accordance with the SIRS guideline. In comparison with healthy control group, IL-33 level, as well as IL-17 and other pro-inflammatory cytokines, was significantly up-regulated in the disease group. This is the first clinical assessment of the correlation between IL-33 level and clinical sepsis. Interestingly, soluble ST2, a decoy receptor by binding IL-33 and inhibiting IL-33/ST2 signaling, was elevated in serums of patients with severe sepsis and correlated with mortality rate [31]. In our mouse sepsis model, IL-33, IL-17 and other pro-inflammatory cytokines were also induced in CLP-treated mice. In the same sepsis model, IL-33 injection was shown to enhance the mouse survival rate, and IL1-RL1 (-/-) mice became more susceptible to CLP challenge [10]. In our study, IL-33 (-/-) mice did have higher mortality rate than WT mice after CLP challenge, supporting the notion that IL-33 protects against polymicrobial sepsis.

The molecular basis underlying the protective role of IL-33 and its signaling against sepsis is still under characterization. Previous study has suggested IL-33 attenuated sepsis by enhancing neutrophil influx to the site of infection [10]. IL-33 preventd the downregulation of CXCR2 and inhibition of chemotaxis induced by TLR4 in mouse and human neutrophils. Another study has proposed IL-33 may reduce the apoptosis of T and B lymphocytes in septic mice by acting on both the death receptor and mitochondria pathways of apoptosis [22]. In our study, we have found neutralizing IL-17 improved sepsis survival rate in IL-33 (-/-) mice. IL-17A could be induced in mice by CLP, and neutralizing IL-17A by antibodies improved survival from $\sim 10 \%$ to nearly $60 \%$ [21]. In contrast, another study showed IL-17R deficient mice exhibited impaired host defense to polymicrobial sepsis,

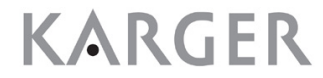


which was associated with a marked reduction in neutrophil recruitment to the lesion site [19]. Therefore, the regulation of IL-17 signaling during sepsis is critical to control infection and resolve systemic inflammation. Our in vitro experiments suggest cross-talk between IL-33 and IL-17 signaling during inflammatory response. We found IL-33 could suppress IL-17-induced pro-inflammatory cytokine productions in MEFs. But IL-33-treated mice still showed enhanced neutrophil recruitment during sepsis [10]. These strongly suggest IL-33 treatment does not inhibit the beneficial effect of IL-17 on controlling the sepsis progression via neutrophil recruitment. Instead, IL-33 may selectively suppress IL-17 signaling, leading to unwanted pro-inflammatory response.

In addition, IL-7 treatment was shown to enhance survival rate of sepsis mice through accelerating neutrophil recruitment and improving bacterial clearance, via activating IL-17 production in $\gamma \delta$ T cells [32]. In our study, we did not detect any change in IL-17 level in mice treated with IL-33. IL-17 is produced in $\gamma \delta$ T cells during sepsis as supported by our TCR $\beta(-/-$ ) model. We also did not observe any change in $\gamma \delta$ T cell number after IL-33 injection in CLP model, therefore IL-33 is probably targeting the signaling components downstream of the IL-17/IL-17R pathway. IL-17-induced pro-inflammatory pathway involves the activation of $\mathrm{NF \kappa B}$ and MAPKs via TAK1 [24]. Our in vitro results showed that IL-33 downregulated IL-17mediated activation of TAK1, IкB $\alpha$ and JNK2, supporting our hypothesis. SOCS3 is a potential key negative physiological regulator of a broad range of pro-inflammatory cytokines and TLR pathways [33]. The expression of SOCS3 gene is induced by various cytokines, including IL-6, IL-10 and IFN- $\gamma[15,34]$. For the first time we hereby report SOCS3 is induced by IL-33 in MEFs, suggesting a novel mechanism utilized by IL-33 signaling to exert anti-inflammatory response during sepsis. IL-17 alone does not activate SOCS3 in our model. Interestingly, IL33 and IL-17 together exhibit synergistic effect to induce SOCS3 with a greater extent than IL-33 alone. We propose that the activation of SOCS3 is responsible for the downregulation of IL-17-mediated pro-inflammatory cytokine productions in CLP model. Such IL-33-mediated SOCS3 activation during sepsis is unlikely to inhibit IL17-induced neutrophil recruitment, as IL-33-treated mice showed enhanced neutrophil influx to the site of infection [10]. Therefore, sepsis treatment targeting the IL-33-SOCS3 axis is unlikely to compromise the host defense against infection. Follow-up studies should be carried out to investigate the level of SOCS3 in sepsis patients, and confirm the activation of SOCS3 in treating sepsis in experimental CLP model. To further characterize the role of SOCS3 in IL-33-mediated sepsis attenuation will inspire new therapeutic strategy for sepsis treatment.

\section{Acknowledgements}

This work was supported by The National Natural Science Foundation of China (81171639) and The Natural science fund of Zhejiang Province (LQ16H150001).

\section{Disclosure Statement}

There is no conflict of interest.

\section{References}




\section{Cellular Physiology Cell Physiol Biochem 2017;42:1961-1972 \begin{tabular}{ll|l} 
and Biochemistry Published onlIne: August 07, 2017 & $\begin{array}{l}\text { (c) } 2017 \text { The Author(s). Published by S. Karger AG, Basel } \\
\text { www.karger.com/cpb }\end{array}$
\end{tabular}}

Lv et al.: New Therapeutic Strategy for Sepsis

5 Shukla P, Rao GM, Pandey G, Sharma S, Mittapelly N, Shegokar R, Mishra PR: Therapeutic interventions in sepsis: current and anticipated pharmacological agents. Br J Pharmacol 2014;171:5011-5031.

-6 Carriere V, Roussel L, Ortega N, Lacorre DA, Americh L, Aguilar L, Bouche G, Girard JP: IL-33, the IL-1-like cytokine ligand for ST2 receptor, is a chromatin-associated nuclear factor in vivo. Proc Natl Acad Sci USA 2007;104:282-287.

7 Liew FY, Pitman NI, McInnes IB: Disease-associated functions of IL-33: the new kid in the IL-1 family. Nat Rev Immunol 2010;10:103-110.

8 De la Fuente M, MacDonald TT, Hermoso MA: The IL-33/ST2 axis: Role in health and disease. Cytokine Growth Factor Rev 2015;26:615-623.

-9 Liew FY, Girard JP, Turnquist HR: Interleukin-33 in health and disease. Nat Rev Immunol 2016;16:676-689.

10 Alves-Filho JC, Sonego F, Souto FO, Freitas A, Verri WA, Jr., Auxiliadora-Martins M, Basile-Filho A, McKenzie AN, Xu D, Cunha FQ, Liew FY: Interleukin-33 attenuates sepsis by enhancing neutrophil influx to the site of infection. Nat Med 2010;16:708-712.

11 Yao Z, Fanslow WC, Seldin MF, Rousseau AM, Painter SL, Comeau MR, Cohen JI, Spriggs MK: Herpesvirus Saimiri encodes a new cytokine, IL-17, which binds to a novel cytokine receptor. Immunity 1995;3:811821.

12 Yan JW, Wang YJ, Peng WJ, Tao JH, Wan YN, Li BZ, Mei B, Chen B, Yao H, Yang GJ, Li XP, Ye DQ Wang J: Therapeutic potential of interleukin-17 in inflammation and autoimmune diseases. Expert Opin Ther Targets 2014;18:29-41.

13 Trengove MC, Ward AC: SOCS proteins in development and disease. Am J Clin Exp Immunol 2013;2:1-29.

14 Baetz A, Frey M, Heeg K, Dalpke AH: Suppressor of cytokine signaling (SOCS) proteins indirectly regulate toll-like receptor signaling in innate immune cells. J Biol Chem 2004;279:54708-54715.

15 Krebs DL, Hilton DJ: SOCS proteins: negative regulators of cytokine signaling. Stem Cells 2001;19:378-387.

16 Fang M, Dai H, Yu G, Gong F: Gene delivery of SOCS3 protects mice from lethal endotoxic shock. Cell Mol Immunol 2005;2:373-377.

17 Bone RC, Balk RA, Cerra FB, Dellinger RP, Fein AM, Knaus WA, Schein RM, Sibbald WJ: Definitions for sepsis and organ failure and guidelines for the use of innovative therapies in sepsis. The ACCP/SCCM Consensus Conference Committee. American College of Chest Physicians/Society of Critical Care Medicine. Chest 1992;101:1644-1655.

18 Kurt-Jones EA, Sandor F, Ortiz Y, Bowen GN, Counter SL, Wang TC, Finberg RW: Use of murine embryonic fibroblasts to define Toll-like receptor activation and specificity. J Endotoxin Res 2004;10:419-424.

19 Freitas A, Alves-Filho JC, Victoni T, Secher T, Lemos HP, Sonego F, Cunha FQ, Ryffel B: IL-17 receptor signaling is required to control polymicrobial sepsis. J Immunol 2009;182:7846-7854.

20 Maravitsa P, Adamopoulou M, Pistiki A, Netea MG, Louis K, Giamarellos-Bourboulis EJ: Systemic overrelease of interleukin-17 in acute kidney injury after septic shock: Clinical and experimental evidence. Immunol Lett 2016;178:68-76.

21 Flierl MA, Rittirsch D, Gao H, Hoesel LM, Nadeau BA, Day DE, Zetoune FS, Sarma JV, Huber-Lang MS, Ferrara JL, Ward PA: Adverse functions of IL-17A in experimental sepsis. FASEB J 2008;22:2198-2205.

-22 Li S, Zhu FX, Zhao XJ, An YZ: The immunoprotective activity of interleukin-33 in mouse model of cecal ligation and puncture-induced sepsis. Immunol Lett 2016;169:1-7.

-23 Xiao Y, Jin J, Chang M, Nakaya M, Hu H, Zou Q, Zhou X, Brittain GC, Cheng X, Sun SC: TPL2 mediates autoimmune inflammation through activation of the TAK1 axis of IL-17 signaling. J Exp Med 2014;211:1689-1702.

24 Gaffen SL: Structure and signalling in the IL-17 receptor family. Nat Rev Immunol 2009;9:556-567.

25 Roark CL, Simonian PL, Fontenot AP, Born WK, O'Brien RL: gammadelta T cells: an important source of IL17. Curr Opin Immunol 2008;20:353-357.

-26 Raifer H, Mahiny AJ, Bollig N, Petermann F, Hellhund A, Kellner K, Guralnik A, Reinhard K, Bothur E, Huber M, Bauer S, Lohning M, Kiss EA, Ganal SC, Diefenbach A, Korn T, Lohoff M: Unlike alphabeta T cells, gammadelta T cells, LTi cells and NKT cells do not require IRF4 for the production of IL-17A and IL-22. Eur J Immunol 2012;42:3189-3201.

27 Zhu J, Duan G, Lang L, Liu Y, Zhu J, Wang H, Liu Y: The Bacterial Component Flagellin Induces Anti-Sepsis Protection Through TLR-5, IL-1RN and VCAN During Polymicrobial Sepsis in Mice. Cell Physiol Biochem 2015;36:446-456. 


\section{Cellular Physiology Cell Physiol Biochem 2017;42:1961-1972

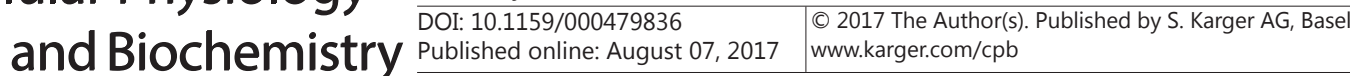

Lv et al.: New Therapeutic Strategy for Sepsis

28 Cheng Y, Wang H, Mao M, Liang C, Zhang Y, Yang D, Wei Z, Gao S, Hu B, Wang L, Cai Q: Escin Increases the Survival Rate of LPS-Induced Septic Mice Through Inhibition of HMGB1 Release from Macrophages. Cell Physiol Biochem 2015;36:1577-1586.

29 Shao Y, He J, Chen F, Cai Y, Zhao J, Lin Y, Yin Z, Tao H, Shao X, Huang P, Yin M, Zhang W, Liu Z, Cui L: Association Study Between Promoter Polymorphisms of ADAM17 and Progression of Sepsis. Cell Physiol Biochem 2016;39:1247-1261.

-30 Oboki K, Ohno T, Kajiwara N, Arae K, Morita H, Ishii A, Nambu A, Abe T, Kiyonari H, Matsumoto K, Sudo K, Okumura K, Saito H, Nakae S: IL-33 is a crucial amplifier of innate rather than acquired immunity. Proc Natl Acad Sci U S A 2010;107:18581-18586.

-31 Hoogerwerf JJ, Tanck MW, van Zoelen MA, Wittebole X, Laterre PF, van der Poll T: Soluble ST2 plasma concentrations predict mortality in severe sepsis. Intensive Care Med 2010;36:630-637.

32 Kasten KR, Prakash PS, Unsinger J, Goetzman HS, England LG, Cave CM, Seitz AP, Mazuski CN, Zhou TT, Morre M, Hotchkiss RS, Hildeman DA, Caldwell CC: Interleukin-7 (IL-7) treatment accelerates neutrophil recruitment through gamma delta T-cell IL-17 production in a murine model of sepsis. Infect Immun 2010;78:4714-4722.

-33 Kubo M, Hanada T, Yoshimura A: Suppressors of cytokine signaling and immunity. Nat Immunol 2003;4:1169-1176.

34 Tamiya T, Kashiwagi I, Takahashi R, Yasukawa H, Yoshimura A: Suppressors of cytokine signaling (SOCS) proteins and JAK/STAT pathways: regulation of T-cell inflammation by SOCS1 and SOCS3. Arterioscler Thromb Vasc Biol 2011;31:980-985. 